\title{
Crossing the exceptional point in a fiber-plasmonic waveguide -INVITED
}

\author{
Alessandro Tuniz ${ }^{1, *}$, Torsten Wieduwilt ${ }^{2}$, and Markus A. Schmidt ${ }^{1}$ \\ ${ }^{1}$ The University of Sydney Nano Institute (Sydney Nano), Institute of Photonics and Optical Science (IPOS), School of Physics, \\ The University of Sydney, NSW 2006, Australia \\ ${ }^{2}$ Leibniz Institute of Photonic Technology (IPHT Jena), Albert-Einstein-Str. 9, 07745 Jena, Germany
}

\begin{abstract}
We experimentally demonstrate a hybrid plasmonic fiber with tuneable Eigenmode interactions near the exceptional point. We experimentally observe a transition through the exceptional point in a fiberplasmonic system: transmission experiments reveal fundamental changes in the underlying Eigenmode interactions as the environmental refractive index is tuned due to a crossing through the plasmonic exceptional point. These results extend the design opportunities for tunable non-Hermitian physics to plasmonic waveguide systems.
\end{abstract}

\section{Introduction}

Photonics is the ideal landscape for investigating nonHermitian physics, where tailored amounts of optical gain and loss can be introduced into a photonic system to unlock otherwise inaccessible Eigenmode topologies [1]. Recent technological advances have shown many farreaching applications [2], which rely on the unique characteristics of the Eigenstates close to exceptional point (EP) - where the system's Eigenvalues coalesce to a degenerate point that is highly sensitive to the environment - including sensitive modal manipulation, topological energy transfer, unidirectional propagation, and polarization conversion. These advances fundamentally rely on the unique characteristics of the eigenstates close to the EP: for example, nanoscale events can be detected by measuring their impact on macroscopic eigenstates [3].

Surface plasmon polaritons (SPP) [4], i.e., propagating surface waves at a metal-dielectric interface, can also be harnessed in the context of non-Hermitian photonics due to their dissipative nature [1]. The high sensitivity of SPPs to refractive index (RI) changes of the environment makes them attractive for sensing applications, leading to intensive research efforts in hybrid dielectric-plasmonic waveguides that can be straightforwardly interfaced with commercial light sources [5]. Such waveguides are inherently nonHermitian and can therefore provide a platform for accessing EP-based functionality. A recent report presented nanostructures with localized plasmon resonances that show discrete frequency spectra [6], but experimental observations of EPs in plasmonic waveguides have so far been limited. Here, we present measurements of the transition across the plasmonic EP in a waveguide system, namely a tuneable hybrid plasmonic fiber [7].

\section{Device Design and Characterization}

A schematic of the experiment is shown in Fig. 1(a). The system is formed by a cylindrical $\mathrm{SiO}_{2}$ waveguide (diameter: $20 \mu \mathrm{m}$ ) coated with a $30 \mathrm{~nm}$ gold nanofilm, surrounded by a liquid of a certain RI $n_{I D}$. Figure $1(b)$ show the properties of the real- and imaginary- parts of the two supported Eigenmodes $\left(\mathrm{EM}_{\mathrm{j}}, \mathrm{j}=1,2\right)$ that can be tuned by changing $\mathrm{n}_{\mathrm{ID}}$ : here the real part of the effective indices either crosses or anti-crosses at a resonant wavelength $\lambda_{R}$, depending on $n_{I D}$. A transition between the two regimes occurs at the EP.

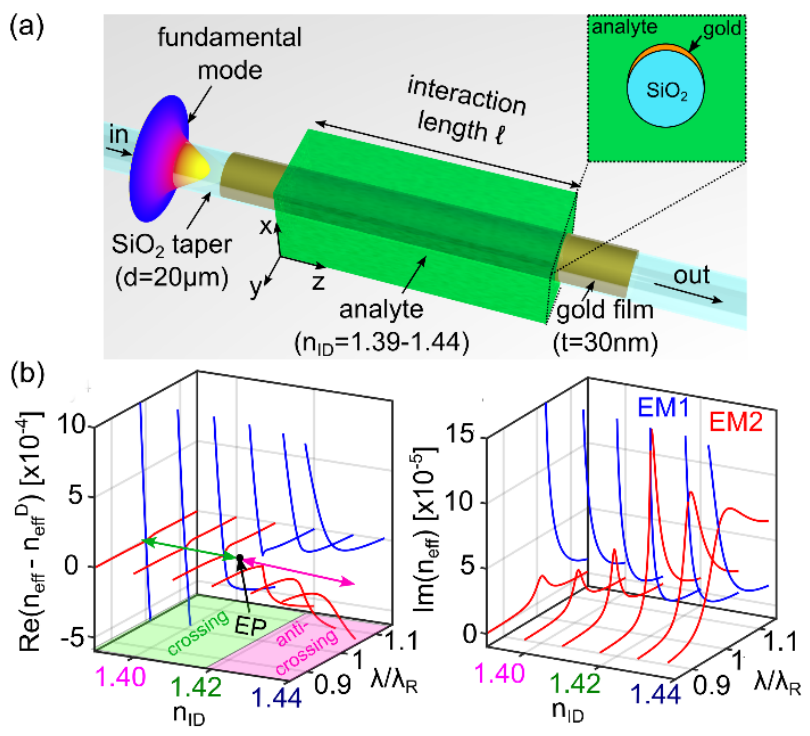

Fig. 1. (a) Hybrid fiber-plasmonic waveguide schematic. A silica fiber coated by a gold nanofilm is immersed in a liquid with RI of $n_{I D}$ and length $\ell$. (b) Calculated spectral distribution of $\operatorname{Re}\left(\mathrm{n}_{\mathrm{eff}, \mathrm{j}}-\mathrm{n}_{\text {eff, } \mathrm{D}}\right)\left(\mathrm{j}=1,2, \mathrm{n}_{\mathrm{eff}, \mathrm{D}}\right.$ : equivalent dielectric waveguide without gold film) and $\operatorname{Im}\left(\mathrm{n}_{\text {eff }}\right)$ for EM1 and EM2 as a function of $\lambda / \lambda_{R}$

\footnotetext{
$\overline{\text { * Corresponding author: alessandro.tuniz@sydney.edu.au }}$
} 
(a) Simulation
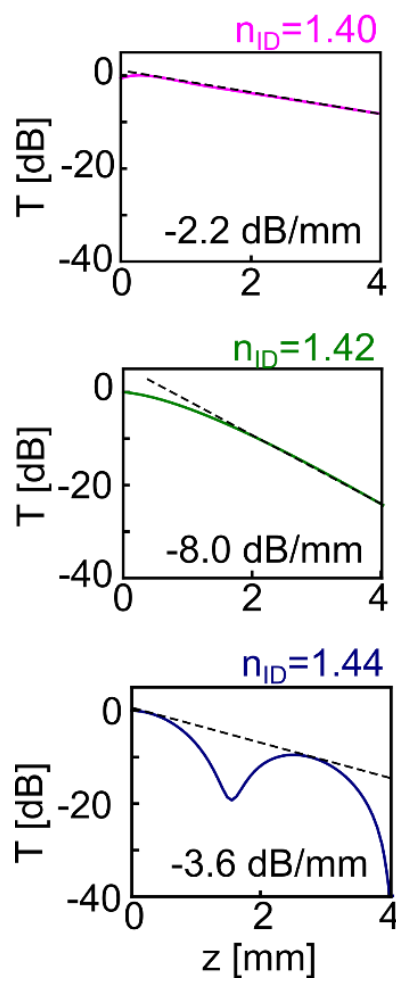

(b) Experiment
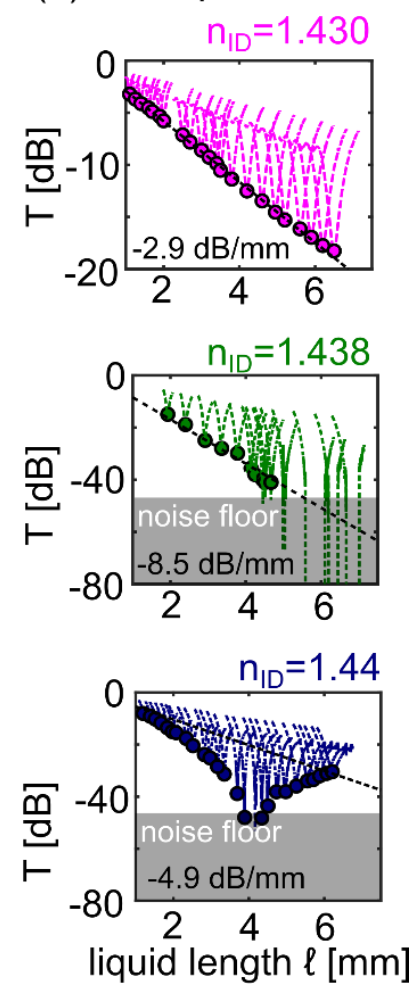

Fig. 2. (a) Calculated transmitted power at three example RIs. (b) Circles: measured on-resonance transmission as a function of $\ell$ for different analytes as labeled, showing the a crossing of the EP. Dashed lines show the full resonance spectrum centered in each transmission minimum (i.e., the horizontal axis of each spectrum is scaled by a factor $\left.\ell / \lambda_{R}[7]\right)$. Dashed lines: fitted loss as labeled

Calculations show that the transition across the EP can be inferred from the transmission properties of this waveguide system by changing $n_{I D}$. Figure 2 (a) shows the calculated transmitted power due to the modal evolution of the two hybrid EMs excited by the fundamental mode of the dielectric waveguide [8] at $\lambda_{R}$. The anti-crossing regime $\mathrm{n}_{\mathrm{ID}}=1.44$ shows an oscillating damped spatial energy distribution, [Fig. 2(a) bottom], while within the crossing regime $\left(\mathrm{n}_{\mathrm{ID}}=1.40\right)$, no such oscillations are observed [Fig. 2(a), top]. At $\mathrm{n}_{\mathrm{ID}}=1.42$ we find that the transmission monotonically decays with the highest overall loss [Fig. 2(a), middle].

We perform a comprehensive series of transmission experiments on the above system [7] - different interaction lengths are realized by liquid columns of different length. Figure 2(b) shows measurements of the on-resonance power $\mathrm{T}$ vs. $\lambda_{\mathrm{R}}$ under different conditions: for $\mathrm{n}_{\mathrm{ID}}=1.440$ we measure a periodic energy exchange between the waveguides (i.e., the anti-crossing regime); a lower $\mathrm{n}_{\mathrm{ID}} \leq 1.438$ yields an exponentially decreasing power with no oscillations (i.e., the crossing regime). Finally, an intermediate refractive index $\mathrm{n}_{\mathrm{ID}}=1.438$ shows no oscillations and the highest loss - a confirmation that the EP was locked via the additional degree of freedom of tuning $\mathrm{n}_{\text {ID. }}$. Since this device operates near the plasmonic cutoff, this sensor has a sensitivity $>50 \mu \mathrm{m} / \mathrm{RIU}$ for $\mathrm{n}_{\text {ID }}>1.43$ [7], which, to the best of our knowledge, is one of the highest values measured for a plasmonic device to date.

\section{Conclusions and Future Directions}

In conclusion, we have presented a plasmonic fiber operating close the exceptional point. Our platform extends the capabilities of tuneable non-Hermitian photonics near the EP to plasmonic waveguides. The results presented here apply to any non-Hermitian waveguide, and immediately provide design tools for future EP-based photonic devices. Preliminary calculations show that this design can be easily modified to operate in the visible regime with a reduced footprint. The unique dispersion characteristics near the EP widen the scope of plasmonics-related applications. For example, this structure can be easily modified for compact operation in the visible regime and at a biologically relevant refractive index $\left(\mathrm{n}_{\text {ID }} \sim 1.33\right)$ : preliminary calculations reveal that a scaled-down version of the above structure in contact with a gold nanofilm possesses an exceptional point at $\mathrm{n}_{\mathrm{ID}}=1.330$, by phase-matching with the short-range surface plasmon polariton [8] at $\lambda \sim$ $560 \mathrm{~nm}$. This device possesses a significantly smaller footprint, both in terms of lateral dimensions (one order of magnitude smaller) and interaction length (four orders of magnitude shorter), while operating in the visible regime. The same principles could be further applied to chip-scale devices with dimensions further reduced by one order of magnitude, e.g. using hybrid plasmonic waveguides modules [9]. These devices could find important applications in convenient, monolithic plasmonic biosensors of single-molecule events [6].

\section{References}

1. M.-A. Miri and A. Alù , Science 363, eaar7709 (2019).

2. Ş.K. Özdemir, S. Rotter, F. Nori, L. Yang, Nat. Mater. 18, 783 (2019).

3. W. Chen, Ş. K. Özdemir, G. Zhao, J. Wiersig, L. Yang, Nature 548, 192 (2017).

4. Lukas Novotny and Bert Hecht, Principles of NanoOptics (Cambridge University Press, Cambridge, England, 2012).

5. T. Wieduwilt, A. Tuniz, S. Linzen, S. Goerke, J. Dellith, U. Hübner, M. A. Schmidt, Sci. Rep. 5, 17060 (2015).

6. J.-H. Park, A. Ndao, W. Cai, L. Hsu, A. Kodigala, T. Lepetit, Y.-H. Lo, B. Kanté, Symmetry-breakinginduced plasmonic exceptional points and nanoscale sensing. Nat. Phys. 16, 462(2020)

7. A. Tuniz, T. Wieduwilt, M. A. Schmidt, Phys. Rev. Lett. 123, 213903 (2019).

8. A. Tuniz and M. A. Schmidt, Optics Express 24, 7507 (2016).

9. A. Tuniz, O. Bickerton, F. J. Diaz, T. Käsebier, E.-B. Kley, S. Kroker, S. Palomba, C. M. de Sterke, Nat. Commun. 11, 2413 (2020) 